\title{
Thermal Assisted UV-Ozone Treatment to Improve Reliability of Ag Nanoparticle Thin Films
}

\author{
Inhwa Lee and Taek-Soo Kim ${ }^{\dagger}$ \\ Department of Mechanical Engineering, KAIST 291 Daehak-ro, Yuseong-gu, Daejeon 305-701, Korea \\ (Received September 13, 2013: Corrected December 30, 2013: Accepted March 2, 2014)
}

\begin{abstract}
We employed UV-Ozone treatment method for the fabrication of dense and highly conductive nanoparticle thin films. We demonstrated the UV-Ozone treatment effect on the silver nanoparticle thin films as a function of time and temperature. The capping layers of nanoparticles were decomposed after UV-Ozone treatment and dense nanoparticle thin films were obtained. Moreover, electrical and mechanical properties of the thin films after UV-Ozone treatment were measured by using resistance measurements under tension in an in-situ tensile tester. The initial resistance of nanoparticle thin films was decreased by $82.6 \%$ with optimized UV-Ozone treatment condition of $150{ }^{\circ} \mathrm{C}$ for 20 minutes.
\end{abstract}

Keywords: Ag nanoparticle, UV-Ozone treatment, thin film, tensile testing

\section{Introduction}

Direct printing method without vacuum deposition process has attracted considerable attention as a new method for fabricating electronic devices such as electrical skin ${ }^{1)}$, displays $^{2)}$ and bendable solar cells. ${ }^{3-4)}$ The direct printing method enables shorter production runs, simpler and lower cost fabrication by exploiting flexible substrate based electronics. Also, thermal deformation problem caused by the high temperature of conventional process can be solved through low temperature process using the lower melting point ${ }^{5)}$ of metal nanoparticle ink.

However, flexible devices are still subjected to large mechanical deformation by stretching, bending and twisting. These problems have resulted in severe performance degradation in practical applications. Porosity and microcracks of metal nanoparticle thin film are pointed as the biggest disadvantages which aggravate the performance degradation. ${ }^{6}$ Therefore, many studies have been conducted to solve these issues through annealing ${ }^{7)}$, plasma $^{8)}$ and UV treatment ${ }^{9)}$ to make dense and reliable metal nanoparticle films. Although those methods mitigate the problems of the nanoparticle thin films described above, more improvements are still necessary.

In this study, we employed thermal assisted UV-Ozone treatment to avoid cracks and voids in silver nanoparticle thin films. It is demonstrated how UV-Ozone treatment and temperature affect reliability of nanoparticle thin films. Electrical resistance curve was used to verify the effect of temperature treatment when implemented with UV-Ozone for silver nanoparticle thin films. It was found that UV-Ozone combined with temperature treatment is much more efficient at improving the reliability of nanoparticle thin film than only UV-Ozone treatment. We present optimal curing condition using UV-Ozone treatment with temperature treatment for reliable nanoparticle thin films. Furthermore, reliability enhancement mechanism of UV-Ozone, temperature treatment, and nanoparticle thin film was introduced.

\section{Experimental}

Polyimide (PI) substrate was spin-coated with nanoparticlebased silver ink (DGP 40LT-15C, Advanced Nano Products, Korea) at $5000 \mathrm{rpm}$ for 30 seconds. The diameter of nanoparticle ranged from 30 to $50 \mathrm{~nm}$ and the silver nanoparticle-ink contained $35 \mathrm{wt} \%$ of metal in methyl alcohol solvent. Then, sintering process was conducted in a convection oven at $150^{\circ} \mathrm{C}$ for 30 minutes to evaporate the solvent. After this initial sintering process, silver nanoparticle films were further treated by UV-Ozone cleaner (Novascan Technologies, Inc., USA) at various temperatures $(50,100$, and $150^{\circ} \mathrm{C}$ ) and time periods (20 and 60 minutes). The

'Corresponding author

E-mail: tskim1@kaist.ac.kr

(C) 2014, The Korean Microelectronics and Packaging Society

This is an Open-Access article distributed under the terms of the Creative Commons Attribution Non-Commercial License(http://creativecommons.org/ licenses/by-nc/3.0) which permits unrestricted non-commercial use, distribution, and reproduction in any medium, provided the original work is properly cited. 
intense ultraviolet light of UV-Ozone cleaner emitted at wave lengths of 185 and $254 \mathrm{~nm}$ and used to improve wettability of the PI surfaces and decompose of organic shells of nanoparticles. ${ }^{10)}$

Tensile test was employed in order to examine the resistance change of silver nanoparticle thin films that underwent UV-Ozone treatment. Cutting plotter (Graphtec FC 8000 ) was used to fabricate $3.0 \times 28 \mathrm{~mm}$ specimens. They were placed under tensile loading with constant displacement rate of $5 \mu \mathrm{m} \mathrm{s}^{-1}$ by high-precision micromechanical test system (Delaminator Adhesion Test System; DTS Company, Menlo Park, CA, USA). Then their resistance was measured with a Keithley 2000 multimeter using a four-wire measurement during the tensile test. From these data, both force-displacement and resistance-time plots were obtained.

Finally, surface morphology of scanning electron microscope (SEM) and focused ion beam (FIB) were conducted in order to characterize surfaces of silver nanoparticle films.

\section{Results and Discussion}

Figure 1 shows a schematic mechanism of UV-Ozone treatment to silver nanoparticle. By attacking contaminants at the molecular level, the organic shells of silver nanoparticles were decomposed.

Figure 2 shows that how nanoparticles reaction changes in relation to the varying durations of UV-Ozone treatment. There was almost no difference in resistance until 10 minutes of UV-Ozone treatment. However, a drastic increase in resistance was observed after 20 minutes of UVOzone treatment. This phenomenon was characterized by SEM images. There was no change on the surface at 5 minutes treatment but small circle spots of $\sim 300 \mathrm{~nm}$ were observed at 10 minutes. Also, the range of the responded area grew bigger and the surface of nanoparticle thin film showed significant change with the increase of time. At 20 and 60 minutes, it is clearly observed that the surface has been covered with a skin layer, and this skin layer is presumably expected to have greatly increased the resistance of nanoparticle thin film.

With these results, we recognized that after UV-Ozone

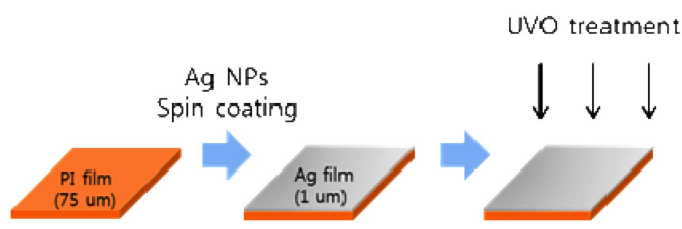

Fig. 1. Schematic process of experiment. (a)
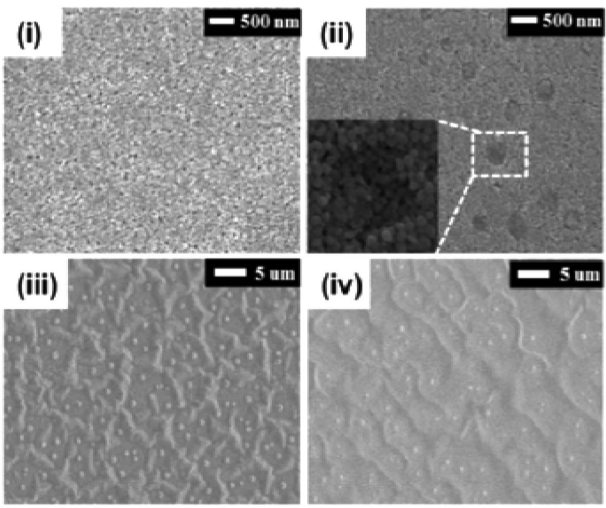

(b)

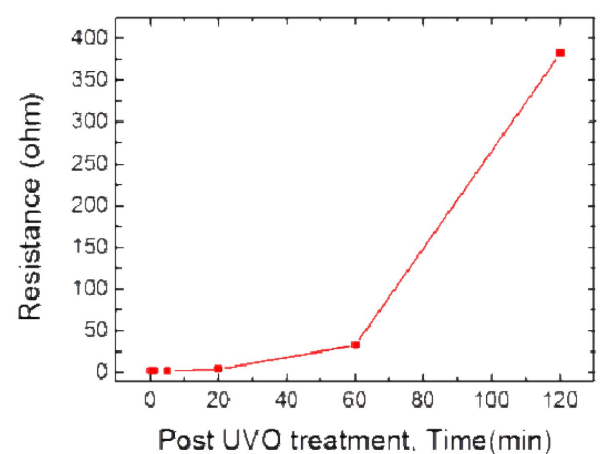

Fig. 2. (a) SEM images of nanoparticle thin film after UV-Ozone treatment for (i) $5 \mathrm{~min}$, (ii) $10 \mathrm{~min}$, (iii) $20 \mathrm{~min}$ and (iv) 60 min. (b) resistance variation with respect to various time conditions.

treatment of 10 minutes and onward time factor contributes to drastic increase in resistance, without affecting surface morphology. On the other hand, the effect of temperature on silver nanoparticle thin film shows a different trend.

Figure 3 shows the temperature effect under UV-Ozone treatment. The period of 20 minutes was fixed in order to investigate the temperature effect. Observing SEM images, the effect of temperature was very minor at $50^{\circ} \mathrm{C}$, but showed significant changes starting at $100^{\circ} \mathrm{C}$, and also on $150^{\circ} \mathrm{C}$. After treatment at $100^{\circ} \mathrm{C}$, the skin layer has partially disappeared. A closer look on this image shows that dark areas are well-annealed silver nanoparticle thin film. Thus $100^{\circ} \mathrm{C}$ seem to be the effective temperature in removing the newly formed layer. At $150^{\circ} \mathrm{C}$, newly formed layer almost disappeared and only well-annealed silver nanoparticle thin film remained intact. From energy dispersive $x$-ray spectroscopy (EDS) analysis (Table 1), it can be proved that silver composition has increased from (a) to (d) in ascending order. These results are in accordance with our observation on gradual deterioration of newly formed layer and capping layers of silver nanoparticles under temperature effect.

Cross-section SEM images of FIB clearly reveal UV- 


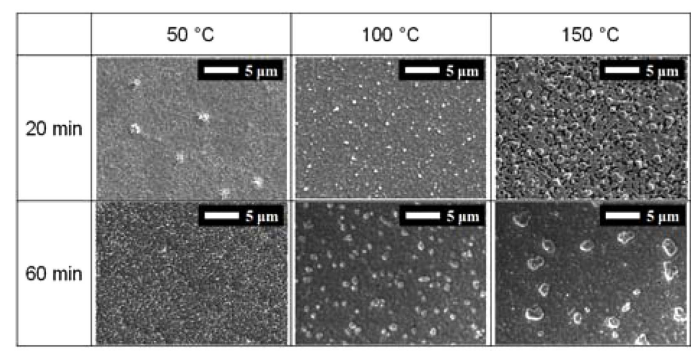

Fig. 3. SEM images with respect to various temperature and time conditions of UV-Ozone treatment.

Table 1. EDS analysis of the surface composition of the silver nanoparticle thin film after UV-Ozone treatment at 50 , 100 and $150^{\circ} \mathrm{C}$ for 20 minutes, respectively.

\begin{tabular}{ccccc}
\hline $\begin{array}{c}\text { Component } \\
\text { (At. \%) }\end{array}$ & $\begin{array}{c}\mathrm{WO} / \\
\mathrm{UVO}\end{array}$ & $\begin{array}{c}\mathrm{UVO} \text { at } \\
50^{\circ} \mathrm{C}\end{array}$ & $\begin{array}{c}\mathrm{UVO} \text { at } \\
100^{\circ} \mathrm{C}\end{array}$ & $\begin{array}{c}\mathrm{UVO} \text { at } \\
150^{\circ} \mathrm{C}\end{array}$ \\
\hline $\mathrm{CK}$ & 43.6 & 35.4 & 30.6 & 40.1 \\
$\mathrm{NK}$ & 02.0 & 08.8 & 05.0 & 08.6 \\
$\mathrm{OK}$ & 05.8 & 26.8 & 31.8 & 13.2 \\
$\mathrm{PtM}$ & 01.6 & 02.0 & 02.4 & 02.1 \\
AgL & $\mathbf{4 6 . 7}$ & $\mathbf{2 6 . 8}$ & $\mathbf{3 0 . 0}$ & $\mathbf{3 5 . 8}$ \\
\hline
\end{tabular}

Ozone treatment reaction mechanism to silver nanoparticles with a function of temperature (Figure 4). We can postulate the reaction mechanism of silver nanoparticle thin films after UV-Ozone treatment in three steps. Firstly, UV was absorbed primarily by the nanoparticles at the top surface forming a skin layer. Secondly, the rest of the unsintered nanoparticles under skin layer began to be sintered due to heat transfer. Lastly, internal vaporization was induced from the thermal decomposition of the organic layers inside the nanoparticles, resulting in film swelling as shown in figure 4(c). Under UV-Ozone treatment alone, Figure 4(b) has been the final observable stage. However, with added temperature effect, we were further able to observe the changes as shown in Figure 4(c-d). This mechanism can be supported by other similar studies that have reported on UVOzone treatment. ${ }^{11-12)}$ Lastly, all organic layers have disappeared and a non-porous uniform metal thin film has been formed as in Figure 4(d).

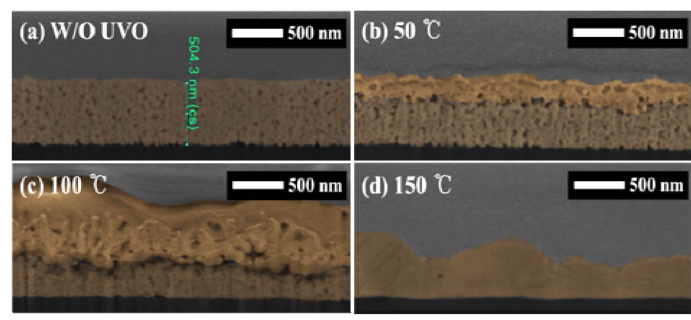

Fig. 4. FIB images of silver nanoparticle thin films: (a) without UV-Ozone treatment, after UV-Ozone treatment at (b) 50 , (c) 100 and (d) $150^{\circ} \mathrm{C}$ for 20 minutes, respectively.
Also, we have conducted tensile test measuring resistance to verify mechanical and electrical properties after UVOzone treatment. Figure 5 shows thin film behavior after 50 $\%$ elongation. On macroscopic scale, large cracks were observed in the thin film of all specimens except for the one that has been cured at $150^{\circ} \mathrm{C}$ for $20 \mathrm{~min}$. At this condition, we can see that there is no distinguishable difference between before and after the tensile loading. Regarding the time effect, there is no huge difference between specimens treated for 20 minutes and 60 minutes except that the latter one's surface seems to be crispier.

Considering the reaction mechanism, it is important to treat the thin film uniformly without an addition of skin layer on top of the surface. At $50^{\circ} \mathrm{C}$, it is found that bottom layer is separated from the skin layer due to the low temperature condition. And at $100^{\circ} \mathrm{C}$, surface is uniform but it is not appropriate because of the dominant swollen organic layer that can be seen in Figure $4(\mathrm{c})$. But at $150^{\circ} \mathrm{C}$ for 20 minutes, we can see that there is no big difference before and after the tensile loading because of the fracture occurs in mesh form.

We also investigated how the organic layer and porosity affect the electrical property of silver nanoparticle thin film. Figure 6 shows resistance variation respect to UV-Ozone treatment temperature. The resistance variation shown in Figure 6(b) is in accordance with the FIB images observed in Figure 4 under temperature variation. The resistance of specimens was maximal at $100^{\circ} \mathrm{C}$ with swollen silver layer of $180 \mathrm{~nm}$ thickness. However when the uniform silver layer was formed at $150^{\circ} \mathrm{C}$, the resistance of specimen significantly decreased. The resistance at $150^{\circ} \mathrm{C}$ is even lower the value measured in original silver nanoparticle thin films. Comparing Figure 2(a) and Figure 6, we can see that temperature is a critical factor to decomposition of organic shells of nanoparticles.

\section{Conclusions}

In conclusion, we demonstrated the UV-Ozone treatment effect on the silver nanoparticle thin films as a function of

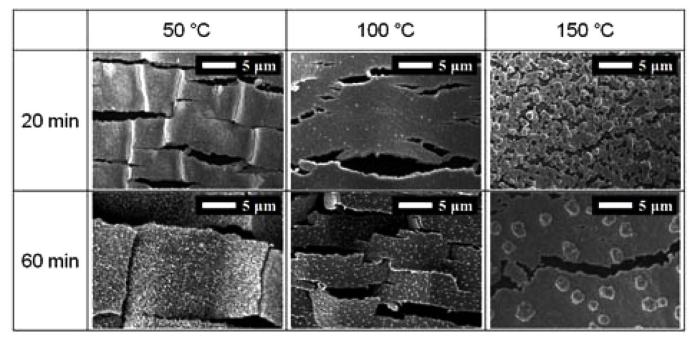

Fig. 5. SEM images with respect to various temperature conditions. 
(a)

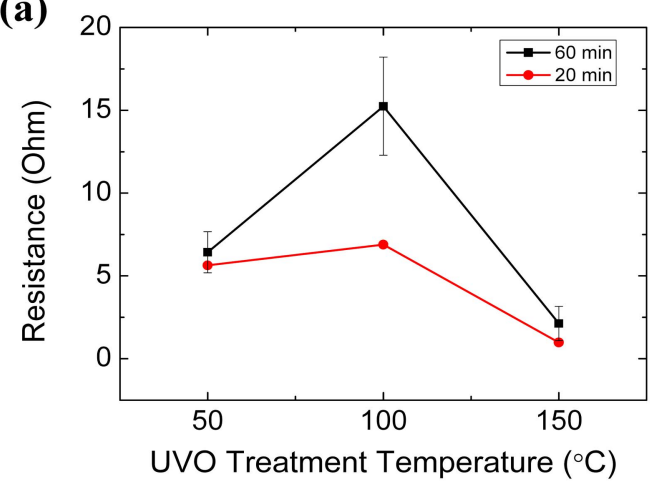

(b)

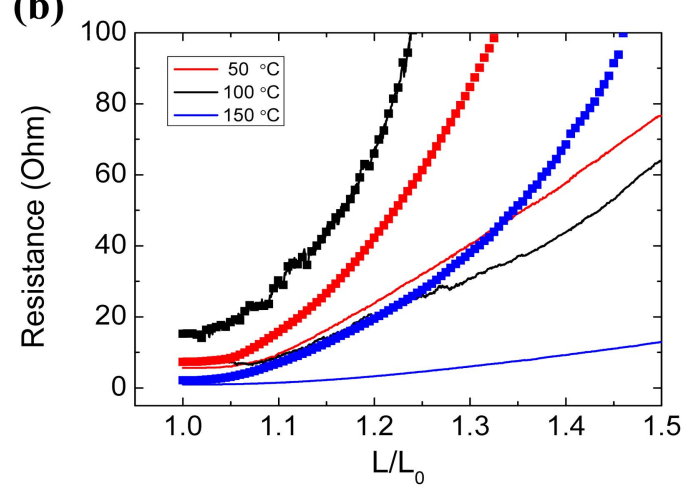

Fig. 6. (a) Resistance variation respect to temperature, (b) Resistance-strain curve of specimens UV-Ozone cured at 50,100 and $150^{\circ} \mathrm{C}$. Results for 20 minutes of treatment are plotted with lines, and for 60 minutes with symbols.

time and temperature. We showed that the skin layer formed during UV-Ozone treatment can be removed by thermally assisted UV-Ozone treatment. UV-Ozone treatment on surface alone is insufficient to anneal the entire nanoparticle thin film because the newly formed layer deters further treatment of the layer underneath it. Simultaneous thermal treatment, however, enables UV-Ozone treatment to be done on entire nanoparticle film; and hence we were able to produce a densely-annealed nanoparticle film under the thermal condition of $150^{\circ} \mathrm{C}$ for 20 minutes. Therefore, optimal UV-Ozone treatment condition should be carefully chosen in order to enhance mechanical and electrical properties in nanoparticle thin films. We believe that present work contributes to the understanding of the mechanism of silver nanoparticle thin films under UV-Ozone treatment.

\section{Acknowledgments}

This work was supported by Basic Science Research Program (2012R1A1A1006072) and the Global Frontier
R\&D Program on Center for Multiscale Energy System (2011-0031569) funded by the National Research Foundation under the Ministry of Science, ICT \& Future Planning, Korea, and also by Graphene Materials and Components Development Program of MOTIE/KEIT (10044412, Development of basic and applied technologies for OLEDs with graphene).

\section{References}

1. K. Takei, T. Takahashi, J. C. Ho, H. Ko, A. G. Gillies, P. W. Leu, R. S. Fearing and A. Javey, "Nanowire active-matrix circuitry for low-voltage macroscale artificial skin", Nature materials, 9(10), 821 (2010).

2. J. Lee, P. Lee, H. Lee, D. Lee, S. S. Lee and S. H. Ko, "Very Long Ag Nanowire Synthesis and Its Application for a Highly Transparent, Conductive and Flexible Metal Electrode Touch Panel", Nanoscale, 4, 6408 (2012).

3. D. J. Lipomi, B. C. K. Tee, M. Vosgueritchian and Z. Bao, "Stretchable organic solar cells", Advanced Materials, 23(15), 1771 (2011).

4. S. H. Kim, J. Y. Choi and H. J. Chang, "Fabrication and Characterization of Organic Solar Cells with Gold Nanoparticles in PEDOT:PSS Hole Transport Layer", J. Microelectron. Packag. Soc., 20(2), 39 (2013).

5. P. Buffat and J. Borel, "Size effect on the melting temperature of gold particles", Physical Review A, 13(6), 2287 (1976).

6. J. H. Lee, N. R. Kim, B. J. Kim, and Y. C. Joo, "Improved mechanical performance of solution-processed MWCNT/Ag nanoparticle composite films with oxygen-pressure-controlled annealing", Carbon, 50(1), 98 (2012).

7. I. Lee, S. Kim, J. Yun, I. Park, and T. S. Kim, "Interfacial toughening of solution processed $\mathrm{Ag}$ nanoparticle thin films by organic residuals", Nanotechnology, 23(48), 485704 (2012).

8. I. Reinhold, C. E. Hendriks, R. Eckardt, J. M. Kranenburg, J. Perelaer, R. R. Baumann, and U. S. Schubert, "Argon plasma sintering of inkjet printed silver tracks on polymer substrates", Journal of Materials Chemistry, 19(21), 3384 (2009).

9. H. S. Kim, S. R. Dhage, D. E. Shim, and H. T. Hahn, "Intense pulsed light sintering of copper nanoink for printed electronics”, Applied Physics A, 97(4), 791 (2009).

10. N. I. Jang and J.-H. Lee, "Effect of PVP Molecular Weight on Size of Sn Nanoparticles Synthesized by Chemical Reduction”, J. Microelectron. Packag. Soc., 18(4), 27 (2011).

11. J. Ryu, H.-S. Kim and H. T. Hahn, "Reactive sintering of copper nanoparticles using intense pulsed light for printed electronics", Journal of Electronic Materials, 40(1), 42 (2011).

12. D. J. Lee, S. H. Park, S. Jang, H. S. Kim, J. H. Oh and Y. W. Song, "Pulsed light sintering characteristics of inkjetprinted nanosilver films on a polymer substrate", Journal of Micromechanics and Microengineering 21(12), 125023 (2011). 